\title{
Characterization of the Pointwise and the Peak Value Behavior of System Approximation under Thresholding
}

\author{
Holger Boche* \\ Technische Universität München \\ Lehrstuhl für Theoretische Informationstechnik \\ Theresienstraße 90, 80333 München, Germany \\ E-mail: boche@tum.de
}

\author{
Ullrich J. Mönich ${ }^{\dagger}$ \\ Massachusetts Institute of Technology \\ Research Laboratory of Electronics \\ 77 Massachusetts Ave., Cambridge, MA 02139, USA \\ E-mail: moenich@mit.edu
}

\begin{abstract}
In this paper we study the system approximation process, which naturally emerges when a stable linear timeinvariant (LTI) system is applied on the Shannon sampling series, for the case that the samples of the signal are disturbed by the non-linear threshold operator, which sets all samples that are below some threshold to zero. We analyze its behavior for signals in the Paley-Wiener space $\mathcal{P} \mathcal{W}_{\pi}^{1}$ of bandlimited signals with absolutely integrable Fourier transform as the threshold tends to zero. We treat the pointwise as well as the global behavior and characterize the systems for which there exist signals such that the approximation error diverges as the threshold tends to zero. We further show that for those systems in a certain topological sense almost all signals lead to divergence and that the divergence can be arbitrarily fast.
\end{abstract}

Keywords-sampling series, linear time invariant system, threshold operator, Paley-Wiener space, divergence speed

\section{INTRODUCTION}

According to Brown's theorem, the Shannon sampling series convergences uniformly on compact subsets of $\mathbb{R}$ for all signals in the Paley-Wiener space $\mathcal{P} \mathcal{W}_{\pi}^{1}$ [1], [2].

Theorem 1 (Brown). For all $f \in \mathcal{P} \mathcal{W}_{\pi}^{1}$ and $\tau>0$ fixed we have

$$
\lim _{N \rightarrow \infty} \max _{t \in[-\tau, \tau]}\left|f(t)-\sum_{k=-N}^{N} f(k) \frac{\sin (\pi(t-k))}{\pi(t-k)}\right|=0 .
$$

The truncation of the series in (1) is done in the domain of the signal $f$ because only the samples $f(k), k=-N, \ldots, N$, are taken into account. In contrast, it is also possible to control the truncation of the series in the codomain of $f$ by considering only the samples $f(k), k \in \mathbb{Z}$, whose absolute value is larger than or equal to some threshold $\delta>0$. This leads to the approximation process

$$
\left(A_{\delta} f\right)(t):=\sum_{\substack{k=-\infty \\|f(k)| \geq \delta}}^{\infty} f(k) \frac{\sin (\pi(t-k))}{\pi(t-k)} .
$$

\footnotetext{
*This work was partly supported by the German Research Foundation (DFG) under grant BO 1734/13-2.

$\uparrow$ Corresponding author. U. Mönich was supported by the German Research Foundation (DFG) under grant MO 2572/1-1.
}

This kind of truncation may appear for example in sensor networks, where, in order to save energy and to reduce interference, the sensors only transmit their measurements to the fusion center if the absolute value of the measured quantity is above some threshold $\delta>0$. Apart from this example, thresholding and quantization, which is closely related to thresholding, are two fundamental operations in digital signal processing because in digital circuits signals can only be represented with a limited resolution and hence must be quantized [3].

In general, $A_{\delta} f$ is only an approximation of $f$. However, since all "important" samples of the signal, i.e., all samples that are larger or equal than $\delta$, are used in the approximation (2), one would expect that $A_{\delta}$ is close to $f$ if $\delta$ is sufficiently small.

In this paper we analyze a more general approximation process

$$
\left(A_{\delta}^{T} f\right)(t):=\left(T A_{\delta} f\right)(t)=\sum_{\substack{k=-\infty \\|f(k)| \geq \delta}}^{\infty} f(k) h_{T}(t-k),
$$

where additionally a linear time-invariant (LTI) system $T$ is present. $h_{T}=T$ sinc is the bandlimited impulse response of the system $T$. In the sensor network example that was given before an additional LTI system enters if we do a further processing of the measured signal. This is for example the case when we extract certain properties of the underlying physical process by using signal processing operations. Clearly, (2) is a special case of (3), which arises if $T$ is the identity operator.

There are two ways to analyze the the approximation processes (2) and (3). One is to study $A_{\delta} f$ and $A_{\delta}^{T} f$ for fixed threshold $\delta$ and varying signals $f$; and the other is to study the behavior of $A_{\delta} f$ and $A_{\delta}^{T} f$ for a fixed signal $f$ as the threshold $\delta$ tends to zero. For fixed threshold $\delta$ the approximation process (2) was analyzed in [4]. It was shown that $A_{\delta}$ is an unbounded operator. However, from this observation we cannot conclude a problematic behavior of $A_{\delta} f$ as $\delta$ tends to zero, because of the non-linearity of $A_{\delta}$. The behavior of the approximation process $A_{\delta} f$ as $\delta$ tends to zero was analyzed in [5]. Surprisingly, the approximation error does not always 
decrease as the threshold $\delta$ tends to zero, i.e., as more and more samples are used for the approximation. Depending on the signal $f \in \mathcal{P} \mathcal{W}_{\pi}^{1}$, the approximation process $\left(A_{\delta} f\right)(t)$, and consequently the approximation error $\left|\left(A_{\delta} f\right)(t)-f(t)\right|$, can diverge unboundedly, even for fixed $t \in \mathbb{R}$, as $\delta$ tends to zero. In contrast to [5] we will study here the situation where an additional stable LTI system is present.

The goal of this paper is to completely understand the effects of the threshold operator on the system approximation process (3). We are interested in the pointwise and the global behavior of the system approximation process $A_{\delta}^{T} f$ as the threshold $\delta$ tends to zero. Further, in the case of divergence, we analyze the structure of the set of divergence creating signals and are able to make a statement about the divergence speed. With the results in this paper we solve three open problems that were formulated in [5, Questions 1-3]. Question 1 deals with the divergence speed of the approximation process $A_{\delta}^{T} f$, Question 2 with the existence of divergence creating signals, and Question 3 with the topological structure of the set of divergence creating signals.

Since the threshold operator is a deterministic operator, we have chosen to treat it deterministically in our analysis. The deterministic analysis is difficult because of the non-linearity of the threshold operator, but it enables us to reveal effects, which cannot be discovered with an additive noise description of the threshold operator. The deterministic approach is in the spirit of [6].

\section{NOTATION}

Let $\hat{f}$ denote the Fourier transform of a function $f$, where $\hat{f}$ is to be understood in the distributional sense. $L^{p}(\mathbb{R}), 1 \leq p<$ $\infty$, is the space of all to the $p$ th power Lebesgue integrable functions on $\mathbb{R}$, with the usual norm $\|\cdot\|_{p}$, and $L^{\infty}(\mathbb{R})$ the space of all functions for which the essential supremum norm $\|\cdot\|_{\infty}$ is finite.

For $\sigma>0$ let $\mathcal{B}_{\sigma}$ be the set of all entire functions $f$ with the property that for all $\epsilon>0$ there exists a constant $C(\epsilon)$ with $|f(z)| \leq C(\epsilon) \exp ((\sigma+\epsilon)|z|)$ for all $z \in \mathbb{C}$. The Bernstein space $\overline{\mathcal{B}}_{\sigma}^{p}$ consists of all functions in $\mathcal{B}_{\sigma}$, whose restriction to the real line is in $L^{p}(\mathbb{R}), 1 \leq p \leq \infty$. A function in $\mathcal{B}_{\sigma}^{p}$ is called bandlimited to $\sigma$. By the Paley-Wiener-Schwartz theorem, the Fourier transform of a function bandlimited to $\sigma$ is supported in $[-\sigma, \sigma]$. For $1 \leq p \leq 2$ the Fourier transformation is defined in the classical and for $p>2$ in the distributional sense.

For $\sigma>0$ and $1 \leq p \leq \infty$ we denote by $\mathcal{P} \mathcal{W}_{\sigma}^{p}$ the PaleyWiener space of functions $f$ with a representation $f(z)=$ $1 /(2 \pi) \int_{-\sigma}^{\sigma} g(\omega) \mathrm{e}^{i z \omega} \mathrm{d} \omega, z \in \mathbb{C}$, for some $g \in L^{p}[-\sigma, \sigma]$. If $f \in \mathcal{P} \mathcal{W}_{\sigma}^{p}$ then $g(\omega)=\hat{f}(\omega)$. The norm for $\mathcal{P} \mathcal{W}_{\sigma}^{p}, 1 \leq p<$ $\infty$, is given by $\|f\|_{\mathcal{P} \mathcal{W}_{\sigma}^{p}}=\left(1 /(2 \pi) \int_{-\sigma}^{\sigma}|\hat{f}(\omega)|^{p} \mathrm{~d} \omega\right)^{1 / p}$.

\section{STABLE LTI SySTEMS}

We briefly review some definitions and facts about stable linear time-invariant (LTI) systems. A linear system $T$ : $\mathcal{P} \mathcal{W}_{\pi}^{1} \rightarrow \mathcal{P} \mathcal{W}_{\pi}^{1}$ is called stable if the operator $T$ is bounded, i.e., if $\|T\|=\sup _{\|f\|_{\mathcal{P} \mathcal{W}_{\pi}^{1} \leq 1}}\|T f\|_{\mathcal{P} \mathcal{W}_{\pi}^{1}}<\infty$. Furthermore, it is called time-invariant if $(T f(\cdot-a))(t)=(T f)(t-a)$ for all $f \in \mathcal{P W}_{\pi}^{1}$ and $t, a \in \mathbb{R}$.
For every stable LTI system $T: \mathcal{P} \mathcal{W}_{\pi}^{1} \rightarrow \mathcal{P} \mathcal{W}_{\pi}^{1}$ there exists exactly one function $\hat{h}_{T} \in L^{\infty}[-\pi, \pi]$ such that

$$
(T f)(t)=\frac{1}{2 \pi} \int_{-\pi}^{\pi} \hat{f}(\omega) \hat{h}_{T}(\omega) \mathrm{e}^{i \omega t} \mathrm{~d} \omega
$$

for all $f \in \mathcal{P} \mathcal{W}_{\pi}^{1}$. Conversely, every function $\hat{h}_{T} \in L^{\infty}[-\pi, \pi]$ defines a stable LTI system $T: \mathcal{P} \mathcal{W}_{\pi}^{1} \rightarrow \mathcal{P} \mathcal{W}_{\pi}^{1}$. We have $h_{T}=T$ sinc, where

$$
\operatorname{sinc}(t)= \begin{cases}\frac{\sin (\pi t)}{\pi t}, & t \neq 0 \\ 1, & t=0 .\end{cases}
$$

Note that $\hat{h}_{T} \in L^{\infty}[-\pi, \pi] \subset L^{2}[-\pi, \pi]$, and consequently $h_{T} \in \mathcal{P} \mathcal{W}_{\pi}^{2}$.

If the samples $\{f(k)\}_{k \in \mathbb{Z}}$ of a signal $f$ are known perfectly, we can use

$$
\sum_{k=-N}^{N} f(k) h_{T}(t-k)
$$

to obtain an approximation of $T f$. The convergence of (5) for $f \in \mathcal{P W}_{\pi}^{1}$ as $N$ goes to infinity is delicate and has to be checked from case. In this paper we analyze the approximation behavior of (3), where the samples of the signals $f \in \mathcal{P} \mathcal{W}_{\pi}^{1}$ are disturbed by the threshold operator.

\section{THE THRESHOLD OPERATOR}

Next, we introduce the threshold operator and discuss some of its basic properties. For complex numbers $z \in \mathbb{C}$, the threshold operator $\kappa_{\delta}, \delta>0$, is defined by

$$
\kappa_{\delta} z= \begin{cases}z & |z| \geq \delta \\ 0 & |z|<\delta\end{cases}
$$

Furthermore, for continuous signals $f: \mathbb{R} \rightarrow \mathbb{C}$, we define the threshold operator $\Theta_{\delta}, \delta>0$, pointwise, i.e., $\left(\Theta_{\delta} f\right)(t)=$ $\kappa_{\delta} f(t), t \in \mathbb{R}$.

In this paper, the threshold operator $\kappa_{\delta}$ is applied on the samples $\{f(k)\}_{k \in \mathbb{Z}}$ of signals $f \in \mathcal{P} \mathcal{W}_{\pi}^{1}$, which gives the disturbed samples $\left\{\kappa_{\delta} f(k)\right\}_{k \in \mathbb{Z}}$. This is, of course, equivalent to applying the threshold operator $\Theta_{\delta}$ on the signal $f$ itself and then taking the samples, i.e., $\left\{\left(\Theta_{\delta} f\right)(k)\right\}_{k \in \mathbb{Z}}$. Then, the resulting samples $\left\{\left(\Theta_{\delta} f\right)(k)\right\}_{k \in \mathbb{Z}}$ are used to build an approximation

$$
\begin{aligned}
\left(A_{\delta}^{T} f\right)(t) & :=\sum_{\substack{k=-\infty \\
\infty}}^{\infty}\left(\Theta_{\delta} f\right)(k) h_{T}(t-k) \\
& =\sum_{\substack{k=-\infty \\
|f(k)| \geq \delta}}^{\infty} f(k) h_{T}(t-k),
\end{aligned}
$$

of the system output $T f$. By $A_{\delta}^{T}$ we denote the operator that maps for to $A_{\delta}^{T} f$ according to (6). If $f \in \mathcal{P} \mathcal{W}_{\pi}^{1}$ we have $\lim _{t \rightarrow \infty} f(t)=0$ by the Riemann-Lebesgue lemma, and it follows that the series in (6) has only finitely many summands, which implies $A_{\delta}^{T} f \in \mathcal{P} \mathcal{W}_{\pi}^{2} \subset \mathcal{P} \mathcal{W}_{\pi}^{1}$. 
The analysis of the approximation processes (6) is difficult, because the operator $A_{\delta}^{T}$ has several properties, which complicate its treatment: 1) For every $\delta>0, A_{\delta}^{T}: \mathcal{P} \mathcal{W}_{\pi}^{1} \rightarrow \mathcal{P} \mathcal{W}_{\pi}^{2}$ is a non-linear operator. 2) For every $\delta>0$, the operator $A_{\delta}^{T}: \mathcal{P} \mathcal{W}_{\pi}^{1} \rightarrow \mathcal{P} \mathcal{W}_{\pi}^{2}$ is discontinuous. 3) For certain $f \in$ $\mathcal{P} \mathcal{W}_{\pi}^{1}$, the operator $A_{\delta}^{T}$ is also discontinuous with respect to $\delta$. In particular the non-linearity leads to the unexpected behavior of the operator $A_{\delta}^{T}$.

\section{Residual SET}

In order to state our main results, we need to introduce the notion of a "residual set".

A subset $G$ of a metric space $X$ is said to be nowhere dense in $X$ if the closure $[G]$ does not contain a non-empty open set of $X . G$ is said to be of the first category (or meager) if $G$ is the countable union of sets each of which is nowhere dense in $X$. $G$ is said to be of the second category (or nonmeager) if is not of the first category. The complement of a set of the first category is called a residual set. Sets of first category may be considered as "small". According to Baire's theorem [7] we have that in a complete metric space, the residual set is dense and a set of the second category. Thus, a residual set is "large" and contains "almost all" elements.

\section{POINTWISE BEHAVIOR}

In this section we analyze the behavior of $\left(A_{\delta}^{T} f\right)(t)$ for fixed $t \in \mathbb{R}$ as the threshold $\delta$ is decreased to zero.

Definition 1. Let $\Phi$ be the set of all continuous, positive, and monotonically decreasing functions $\phi$ defined on $(0,1]$ that satisfy $\lim _{\delta \rightarrow 0} \phi(\delta)=\infty$ and $\phi(\delta) \geq 1$ for all $0<\delta \leq 1$.

For fixed $t \in \mathbb{R}$, we want to characterize the stable LTI systems $T$ for which the set

$$
\mathcal{D}_{1}(T, t, \phi):=\left\{f \in \mathcal{P} \mathcal{W}_{\pi}^{1}: \limsup _{\delta \rightarrow 0} \frac{\left|\left(A_{\delta}^{T} f\right)(t)\right|}{\phi(\delta)}=\infty\right\}
$$

is non-empty, and, in the case where $\mathcal{D}_{1}(T, t, \phi)$ is non-empty, we are interested in structure of this set. For a given LTI system $T$ and time instant $t \in \mathbb{R}$ the set $\mathcal{D}_{1}(T, t, \phi)$ contains all signals $f \in \mathcal{P} \mathcal{W}_{\pi}^{1}$ for which $\left(A_{\delta}^{T} f\right)(t)$ diverges, i.e., all signals for which the approximation process diverges pointwise. The function $\phi \in \Phi$ is introduced in the above expression in order to describe the divergence speed of $\left(A_{\delta}^{T} f\right)(t)$.

Theorem 2. Let $T$ be an stable LTI system, $t \in \mathbb{R}$, and $\phi \in \Phi$. Then we have $\mathcal{D}_{1}(T, t, \phi) \neq \emptyset$ if and only if $\sum_{k=-\infty}^{\infty} \mid h_{T}(t-$ $k) \mid=\infty$. Further, if $\sum_{k=-\infty}^{\infty}\left|h_{T}(t-k)\right|=\infty$ then $\mathcal{D}_{1}(T, t, \phi)$ is a residual set.

Theorem 2 shows that $\mathcal{D}_{1}(T, t, \phi)$ is a residual set if and only if

$$
\sum_{k=-\infty}^{\infty}\left|h_{T}(t-k)\right|=\infty
$$

This means that in a topological sense almost all signals $f \in$ $\mathcal{P} \mathcal{W}_{\pi}^{1}$ lead to a divergent approximation process if (7) is true. Moreover, since the condition (7) does not depend on $\phi$, it follows that in the case of divergence $\mathcal{D}_{1}(T, t, \phi)$ is a residual set for all functions $\phi \in \Phi$. That is, the divergence speed can be arbitrarily fast. Theorem 2 solves the Questions 1-3 from [5]. It is interesting to note that (7) is a necessary and sufficient condition. That is, Theorem 2 gives us a complete characterization of the systems $T$ that can be approximated by using the approximation process $A_{\delta}^{T}$.

The proof of Theorem 2 is technically rather involved and omitted due to space constraints.

Next, we want to apply Theorem 2. For the LTI system $T=$ $I d$, where $I d$ denotes the identity operator, we have $h_{T}=$ sinc and thus obtain, as a special case of (6), the sampling series

$$
\left(A_{\delta} f\right)(t)=\left(A_{\delta}^{I d} f\right)(t)=\sum_{\substack{k=-\infty \\|f(k)| \geq \delta}}^{\infty} f(k) \frac{\sin (\pi(t-k))}{\pi(t-k)},
$$

which is the Shannon sampling series that uses only the samples that are larger than or equal to the threshold $\delta$. Since $\sum_{k=-\infty}^{\infty}|\operatorname{sinc}(t-k)|=\infty$ for all $t \in \mathbb{R} \backslash \mathbb{Z}$, we obtain the next corollary as an immediate consequence of Theorem 2 .

Corollary 1. Let $t \in \mathbb{R} \backslash \mathbb{Z}$ and $\phi \in \Phi$. Then

$$
\left\{f \in \mathcal{P} \mathcal{W}_{\pi}^{1}: \limsup _{\delta \rightarrow 0} \frac{\left|\left(A_{\delta} f\right)(t)\right|}{\phi(\delta)}=\infty\right\}
$$

is a residual set.

Corollary 1 shows the difference between the Shannon sampling series

$$
\sum_{k=-N}^{N} f(k) \frac{\sin (\pi(t-k))}{\pi(t-k)}
$$

where the truncation is controlled in the domain of the signal, and our approximation process $A_{\delta} f$, where the truncation is controlled in the codomain of $f$. The Shannon sampling series is locally uniformly convergent for all $f \in \mathcal{P} \mathcal{W}_{\pi}^{1}$ according to Brown's theorem, whereas $A_{\delta} f$ can be divergent for certain signals $f \in \mathcal{P} \mathcal{W}_{\pi}^{1}$.

In particular, Corollary 1 shows that for fixed $t \in \mathbb{R} \backslash \mathbb{Z}$ there exists a signal $f \in \mathcal{P} \mathcal{W}_{\pi}^{1}$ such that

$$
\limsup _{\delta \rightarrow 0} \frac{\left|\left(A_{\delta} f\right)(t)\right|}{\phi(\delta)}=\infty
$$

The next corollary strengthens this assertion. It states that there exists a universal signal $f \in \mathcal{P} \mathcal{W}_{\pi}^{1}$ such that we have divergence as in (8) for all $t \in \mathbb{R} \backslash \mathbb{Z}$.

Corollary 2. Let $\phi \in \Phi$. Then

$$
\left\{f \in \mathcal{P} \mathcal{W}_{\pi}^{1}: \limsup _{\delta \rightarrow 0} \frac{\left|\left(A_{\delta} f\right)(t)\right|}{\phi(\delta)}=\infty \text { for all } t \in \mathbb{R} \backslash \mathbb{Z}\right\}
$$

is a residual set.

Proof: Let $t_{1} \in \mathbb{R} \backslash \mathbb{Z}$ and $\phi \in \Phi$ be arbitrary but fixed. According to Corollary 1, there exists a residual set $G \subset \mathcal{P} \mathcal{W}_{\pi}^{1}$ such that

$$
\limsup _{\delta \rightarrow 0} \frac{\left|\left(A_{\delta} f\right)\left(t_{1}\right)\right|}{\phi(\delta)}=\infty
$$


for all $f \in G$. We further have, for $t_{2} \in \mathbb{R} \backslash \mathbb{Z}, 0<\delta<1$, and $f \in \mathcal{P} \mathcal{W}_{\pi}^{1}$ that

$$
\begin{aligned}
& \left|\frac{\left(A_{\delta} f\right)\left(t_{1}\right)}{\phi(\delta) \sin \left(\pi t_{1}\right)}-\frac{\left(A_{\delta} f\right)\left(t_{2}\right)}{\phi(\delta) \sin \left(\pi t_{2}\right)}\right| \\
& =\frac{1}{\phi(\delta) \pi}\left|\sum_{\substack{k=-\infty \\
|f(k)| \geq \delta}}^{\infty} f(k) \frac{(-1)^{k}}{t_{1}-k}-\sum_{\substack{k=-\infty \\
|f(k)| \geq \delta}}^{\infty} f(k) \frac{(-1)^{k}}{t_{2}-k}\right| \\
& \leq \frac{\|f\|_{\mathcal{P} \mathcal{W}_{\pi}^{1}}}{\pi} \sum_{\substack{k=-\infty \\
|f(k)| \geq \delta}}^{\infty} \frac{\left|t_{2}-t_{1}\right|}{\left|t_{1}-k\right|\left|t_{2}-k\right|}=: C_{1}\left(t_{1}, t_{2}, f\right),
\end{aligned}
$$

where $C_{1}\left(t_{1}, t_{2}, f\right)<\infty$ is a constant that depends only on $t_{1}, t_{2}$, and $f$. It follows that

$$
\frac{\left|\left(A_{\delta} f\right)\left(t_{2}\right)\right|}{\phi(\delta)} \geq \frac{\left|\left(A_{\delta} f\right)\left(t_{1}\right)\right|}{\phi(\delta)}\left|\frac{\sin \left(\pi t_{2}\right)}{\sin \left(\pi t_{1}\right)}\right|-C_{2}\left(t, t_{2}, f\right)
$$

for all $t_{2} \in \mathbb{R} \backslash \mathbb{Z}, 0<\delta<1, f \in G$. Taking the limit superior on both sides of (9) gives

$$
\limsup _{\delta \rightarrow 0} \frac{\left|\left(A_{\delta} f\right)\left(t_{2}\right)\right|}{\phi(\delta)}=\infty
$$

for all $t_{2} \in \mathbb{R} \backslash \mathbb{Z}$ and all $f \in G$.

\section{GLOBAL BEHAVIOR}

In this section we study the behavior of $\left\|A_{\delta}^{T} f\right\|_{\infty}$, i.e., the $L^{\infty}$-norm of the approximation process, as the threshold $\delta$ is decreased to zero. The set of interest in this case is

$$
\mathcal{D}_{1}^{\infty}(T, \phi)=\left\{f \in \mathcal{P} \mathcal{W}_{\pi}^{1}: \limsup _{\delta \rightarrow 0} \frac{\left\|A_{\delta}^{T} f\right\|_{\infty}}{\phi(\delta)}=\infty\right\}
$$

Theorem 3. Let $T$ be a stable LTI system and $\phi \in \Phi$. Then we have $\mathcal{D}_{1}^{\infty}(T, \phi) \neq \emptyset$ if and only if $h_{T} \notin \mathcal{B}_{\pi}^{1}$. Further, if $h_{T} \notin \mathcal{B}_{\pi}^{1}$ then $\mathcal{D}_{1}^{\infty}(T, \phi)$ is a residual set.

For the proof of Theorem 3 we need the following lemma.

Lemma 1. Let $h_{T} \in \mathcal{B}_{\pi}$. If

$$
\sum_{k=-\infty}^{\infty}\left|h_{T}(k)\right|<\infty
$$

and

$$
\sum_{k=-\infty}^{\infty}\left|h_{T}\left(k+\frac{1}{2}\right)\right|<\infty
$$

then we have $h_{T} \in \mathcal{B}_{\pi}^{1}$.

Lemma 1 follows directly from the fact that conditions (10) and (11) together correspond to oversampling with oversampling factor 2 .
Proof of Theorem 3: Let $\phi \in \Phi$ be arbitrary but fixed.

We prove the " $\Rightarrow$ " direction of the if and only if assertion by showing that $h_{T} \in \mathcal{B}_{\pi}^{1}$ implies $\mathcal{D}_{1}^{\infty}(T, \phi)=\emptyset$. Thus, let $T$ be a stable LTI system such that $h_{T} \in \mathcal{B}_{\pi}^{1}$. For all $\delta>0$ and $f \in \mathcal{P} \mathcal{W}_{\pi}^{1}$ we have

$$
\begin{aligned}
\left|\left(A_{\delta}^{T} f\right)(t)\right| & \leq \sum_{\substack{k=-\infty \\
|f(k)| \geq \delta}}^{\infty}\left|f(k) h_{T}(t-k)\right| \\
& \leq\|f\|_{\mathcal{P} \mathcal{W}_{\pi}^{1}} \sum_{k=-\infty}^{\infty}\left|h_{T}(t-k)\right| .
\end{aligned}
$$

It follows, using Nikol'skiı̌'s inequality [8, p. 49], that

$$
\begin{aligned}
\left\|A_{\delta}^{T} f\right\|_{\infty} & \leq\|f\|_{\mathcal{P} \mathcal{W}_{\pi}^{1}} \sup _{t \in \mathbb{R}} \sum_{k=-\infty}^{\infty}\left|h_{T}(t-k)\right| \\
& \leq\|f\|_{\mathcal{P} \mathcal{W}_{\pi}^{1}}\left\|h_{T}\right\|_{1},
\end{aligned}
$$

which implies $\mathcal{D}_{1}^{\infty}(T, \phi)=\emptyset$.

Next, we prove the second assertion of the theorem, i.e., that $\mathcal{D}_{1}^{\infty}(T, \phi)$ is a residual set if $h_{T} \notin \mathcal{B}_{\pi}^{1}$. This also proves the " $\Leftarrow$ " direction of the if and only if assertion. Let the stable LTI system $T$ be such that $h_{T} \notin \mathcal{B}_{\pi}^{1}$. Then we have

$$
\sum_{k=-\infty}^{\infty}\left|h_{T}(k)\right|=\infty
$$

or

$$
\sum_{k=-\infty}^{\infty}\left|h_{T}\left(k+\frac{1}{2}\right)\right|=\infty,
$$

according to Lemma 1. From Theorem 2 it follows that $\mathcal{D}_{1}(T, 0, \phi)$ or $\mathcal{D}_{1}(T, 1 / 2, \phi)$ is a residual set, which in turn implies that $\mathcal{D}_{1}^{\infty}(T, \phi)$ is a residual set.

The proof of Theorem 3 has also revealed the following corollary.

Corollary 3. Let $T$ be a stable LTI system and $\phi \in \Phi$. Then we have $\mathcal{D}_{1}^{\infty}(T, \phi) \neq \emptyset$ if and only if

$$
\sum_{k=-\infty}^{\infty}\left|h_{T}(k)\right|=\infty \quad \text { or } \quad \sum_{k=-\infty}^{\infty}\left|h_{T}\left(k+\frac{1}{2}\right)\right|=\infty .
$$

Moreover, if $\mathcal{D}_{1}^{\infty}(T, \phi) \neq \emptyset$ then $\mathcal{D}_{1}^{\infty}(T, \phi)$ is a residual set.

\section{DISCUSSION}

In this paper we studied the system approximation process under thresholding and solved three open problems that were posed in [5, Questions 1-3]. The results in this paper illustrate that the threshold operator leads to completely new phenomena in the approximation of stable LTI systems.

Corollary 3 and Theorem 2 show the significant difference between the approximation behavior of $A_{\delta}^{T}$ and the approximation behavior of the Shannon sampling series

$$
\left(S_{N} f\right)(t):=\sum_{k=-N}^{N} f(k) \frac{\sin (\pi(t-k))}{\pi(t-k)},
$$

which is described by Brown's theorem. Although the $L^{\infty}$ norm of the Shannon sampling series $\left\|S_{N} f\right\|_{\infty}$ diverges for 
certain signals in $\mathcal{P} \mathcal{W}_{\pi}^{1}$, we still have, for fixed $t \in \mathbb{R}$, convergence for all signals in $\mathcal{P} \mathcal{W}_{\pi}^{1}$. In contrast, the divergence of the $L^{\infty}$-norm of $A_{\delta}^{T} f$ for one signal $f \in \mathcal{P} \mathcal{W}_{\pi}^{1}$ results in the divergence of $\left(A_{\delta}^{T} f\right)(t)$ for $t=0$ or $t=1 / 2$ and all signals from a residual set.

We gave a complete characterization of the stable LTI systems that can be approximated by the approximation process $A_{\delta}^{T}$. For the approximation process (5) such a complete characterization is still unknown, although the operators in this problem are linear.

As for the divergence speed, we have the following difference. For the Shannon sampling series it can be shown that there exists a constant $C_{3}>0$ such that for all $f \in \mathcal{P} \mathcal{W}_{\pi}^{1}$ we have $\left\|S_{N} f\right\|_{\infty} \leq C_{3} \log (N+1)\|f\|_{\mathcal{P} \mathcal{W}^{1}}$ for all $N \in \mathbb{N}$, i.e., the growth speed of $\left\|S_{N} f\right\|_{\infty}$ is bounded above and cannot be arbitrarily fast. This is contrast to the approximation process $A_{\delta}^{T} f$ where the pointwise as well as the global divergence can be arbitrarily fast, as Theorems 2 and 3 have shown.

Thresholding is closely related to quantization, because the mid-tread quantization operator sets all small signal values to zero, like the threshold operator. Hence, we conjecture that similar results as those which where obtained in this paper are also valid for the quantization operator.

Greedy approximation [9], [10] is a topic which seems to be related to the approximation with thresholding that is studied in this paper. However, in greedy approximations the truncation is usually performed in the frequency domain and divergence in the frequency domain does not always translate to divergence in the time domain. For example, it is easy to construct a sequence of $\mathcal{P} \mathcal{W}_{\pi}^{2}$-signals $\left\{f_{n}\right\}_{n \in \mathbb{N}}$ with uniformly bounded $\mathcal{P} \mathcal{W}_{\pi}^{2}$-norm, for which the corresponding sequence of Fourier transforms $\left\{\hat{f}_{n}\right\}_{n \in \mathbb{N}}$ diverges everywhere in $[-\pi, \pi]$. Further, the uniform boundedness of the $\mathcal{P} \mathcal{W}_{\pi}^{2}$-norm implies the uniform boundedness of the $L^{\infty}(\mathbb{R})$-norm. Hence, the results in greedy approximations cannot be simply transfered to our problem.
In general, oversampling is known to improving the convergence behavior of approximation processes. However, in [11] it was shown that, for certain stable LTI systems, oversampling cannot resolve the divergence of the approximation process with thresholding. It is an interesting open problem to characterize the systems that can be stably approximated if oversampling is used in the approximation process.

\section{REFERENCES}

[1] J. L. Brown, Jr., "On the error in reconstructing a non-bandlimited function by means of the bandpass sampling theorem," Journal of Mathematical Analysis and Applications, vol. 18, pp. 75-84, 1967, Erratum, ibid, vol. 21, 1968, p. 699.

[2] P. L. Butzer, W. Splettstößer, and R. L. Stens, "The sampling theorem and linear prediction in signal analysis," Jahresbericht der Deutschen Mathematiker-Vereinigung, vol. 90, no. 1, pp. 1-70, Jan. 1988.

[3] Robert M. Gray and David L. Neuhoff, "Quantization," IEEE Transactions on Information Theory, vol. 44, no. 6, pp. 2325-2383, Oct. 1998.

[4] Holger Boche and Ullrich J. Mönich, "Behavior of the quantization operator for bandlimited, nonoversampled signals," IEEE Transactions on Information Theory, vol. 56, no. 5, pp. 2433-2440, May 2010.

[5] Holger Boche and Ullrich J. Mönich, "Unboundedness of thresholding and quantization for bandlimited signals," Signal Processing, vol. 92, no. 12, pp. 2821-2829, Dec. 2012.

[6] P. L. Butzer and W. Splettstößer, "On quantization, truncation and jitter errors in the sampling theorem and its generalizations," Signal Processing, vol. 2, no. 2, pp. 101-112, Apr. 1980.

[7] Kôaku Yosida, Functional Analysis, Springer-Verlag, 1971.

[8] John R. Higgins, Sampling Theory in Fourier and Signal Analysis Foundations, Oxford University Press, 1996.

[9] T. W. Körner, "Decreasing rearranged Fourier series," Journal of Fourier Analysis and Applications, vol. 5, pp. 1-19, Nov. 1999.

[10] V. N. Temlyakov, "Greedy expansions in Banach spaces," Advances in Computational Mathematics, vol. 26, no. 4, pp. 431-449, May 2007.

[11] Holger Boche and Ullrich J. Mönich, "An impossibility result for linear signal processing under thresholding," IEEE Transactions on Signal Processing, vol. 58, no. 3, pp. 1082-1094, Mar. 2010. 